\title{
Equation of State for Thermodynamic Properties of Fluids*
}

\author{
Robert D. Goodwin ** \\ Institute for Basic Standards, National Bureau of Standards, Boulder, Colo. 80302
}

September 6, 1974

\begin{abstract}
This equation of state was developed from PVT compressibility data on methane and ethane. The highly-constrained form originates on a given liquid-vapor coexistence boundary (described by equations for the vapor pressures and the orthobaric densities). It then requires only five least-squares coefficients, and ensures a qualitatively correct behavior of the $P(\rho, T)$ surface and of its derivatives, especially about the critical point. This nonanalytic equation yields a maximum in the specific heats $C_{v}(\rho, T)$ at the critical point.
\end{abstract}

Key words: Coexistence boundary; critical point; ethane; equation of state; fluids; methane; orthobaric densities; specific heats; vapor pressures.

\section{Symbols and Units}

Subscripts $\underline{\mathrm{c}}$ and $\underline{\mathrm{t}}$ refer to critical and liquid triple points

Subscript $\underline{\sigma}$ refers to liquid-vapor coexistence

$\alpha, b, \delta \quad$ nonlinear constants in the equation of state

$B(\rho)$, density-dependent coefficients in the equa-

$C(\rho) \quad$ tion of state

$\operatorname{C\sigma }(T), \quad$ molal heat capacity for saturated liquid

$C_{v}^{\circ}(T)$

$C_{v}(\rho, T)$, molal heat capacity for ideal gas states

$C_{p}(\rho, T)$, molal heat capacity at constant pressure

$d$,

$J$,

$l$,

mol,

$P$,

$P$
$R$
$\rho$

$P_{\sigma}(\rho)$,

$R$,

$\rho$,

$\sigma$,

$T$,

$T \sigma(\rho)$,

$\theta(\rho)$,

$u(\rho, T)$,

density, $\mathrm{mol} / \mathrm{l}$

the Joule, $1 \mathrm{~N}-\mathrm{m}$

the liter, $10^{-3} \mathrm{~m}^{3}$

$16.043 \mathrm{~g}$ of $\mathrm{CH}_{4} ; 30.070 \mathrm{~g}$ of $\mathrm{C}_{2} \mathrm{H}_{6},\left(\mathrm{C}^{12}\right.$ scale)

pressure in bars, 1 bar $=10^{5} \mathrm{~N} / \mathrm{m}^{2},(1$

at $\mathrm{m}=1.01325 \mathrm{bar}$ )

the vapor pressure, bar

the gas constant, $8.31434(\mathrm{~J} / \mathrm{mol}) / \mathrm{K}$

$d / d_{t}$, density reduced at the liquid triple point

$d / d_{c}$, density reduced at the critical point

temperautre, K, (IPTS-1968)

temperature at liquid-vapor coexistence

defined locus of temperatures, figure 3

$T / T_{\sigma}(\rho)$, temperature reduced at coexistence

$v, \quad \mathrm{l} / d$, molal volume, $\mathrm{l} / \mathrm{mol}$

$W(\rho, T)$, the speed of sound

$x(T), \quad T / T_{c}$, temperature reduced at the critical point

$x_{\sigma}(\rho), \quad T_{\sigma}(\rho) / T_{c}$, reduced temperature at coexistence

$Z(P, \rho, T) \quad P v / R T$, the "compressibility factor"

\footnotetext{
*This work at the National Bureau of Standards was supported by The American Gas Association, 1515 Wilson Boulevard, Arlington, Va. 22209.

** Cryogenics Division, National Bureau of Standards, Boulder, Colo. 80302.
}

\section{Introduction}

A problem of importance for the natural gas industry is the prediction of thermodynamic properties of liquefied, multicomponent mixtures. For the wide range of compositions encountered, it may be necessary to utilize accurate properties of the pure components. We therefore believe that it will be helpful to have a relatively simple and rational equation of state of identical form for each component, such as the equation of state described below.

This equation originates on a given, liquid-vapor coexistence boundary, thus eliminating the longstanding problem of consistency between equations of state and this independently-derived envelope [26]. It ensures a maximum in the specific heats $C_{v}(\rho, T)$ approaching the critical point, qualitatively consistent with experimental behavior near, but not necessarily exactly at this pole [16], and it has only five arbitrary, least-squares coefficients. Experimental compressibility data for methane and ethane have been used at densities to the triple-point liquid density, temperatures to twice the critical, and pressures to 350 bar or greater.

Our objective in the present report is to give a concise description of this new type of equation of state which, with full documentation, has been presented in previous publications [14, 15].

For methane we have shown in [14] that an equation similar to that described below yields calculated specific heats, $C_{\sigma}(T), C_{v}(\rho, T), C_{p}(\rho, T)$, and speeds of sound in acceptable agreement with experimental data, without any weighting of the equation of state to those data. In a current report on ethane [15] we compute provisional thermodynamic and related properties by means of the simpler equation of state (1) described herein.

Symbols and units used here are given in a List. Fixed-point values from $[14,15]$ are given in table 1 . 
For equation of state (1) the gas constant is $R \equiv(0.0831434) \cdot\left(d_{t}\right) \mathrm{bar} / \mathrm{K}$, consistent with use of the dimensionless density, $\rho \equiv d / d_{t}$.

TABLE 1. Fixed-point values from $[14,15]$

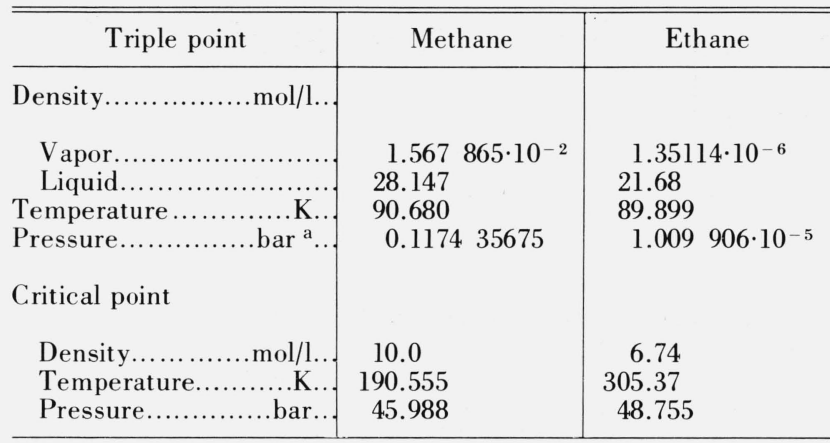

${ }^{a}$ Precision required for vapor-pressure equation.

Various methods for utilizing the equations of state of pure components to derive properties of mixtures are described in a number of recent publications, e.g., $[1,4 \mathrm{a}, 17,18,19,20,21,21 \mathrm{a}, 22,23,24,271$.

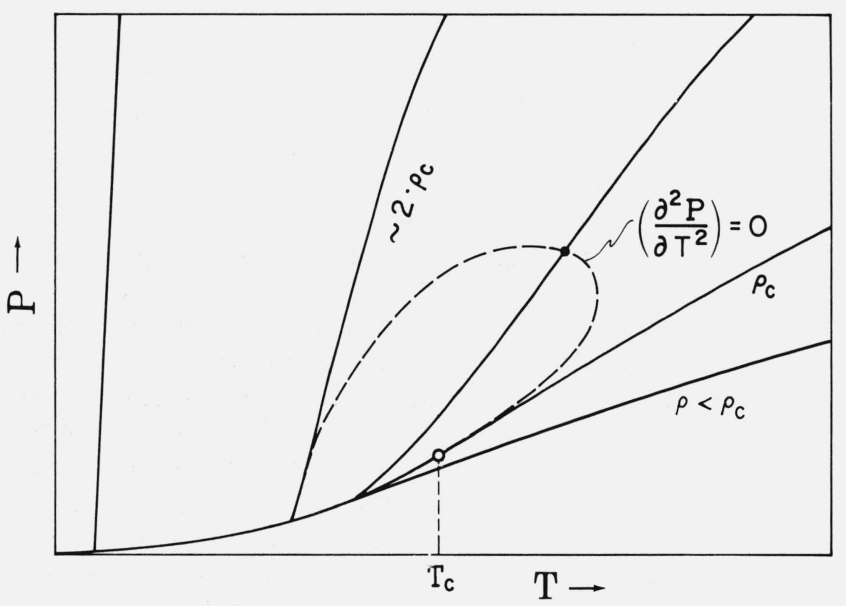

FIGURE 1. The locus of isochore inflection points.

\section{The Equation of State}

The $P(\rho, T)$ surface and equations of state are described in several reports, e.g., $[6,7,10,12,14,15$, $20,24,28]$. Figure 1 shows the qualitative behavior of isochores as indicated by Rowlinson [20], needed to give a calculated maximum in $C_{v}(\rho, T)$ at the critical point via the isothermal computation-

$$
\Delta C_{v}=-T \cdot \int_{0}^{\rho}\left(\partial^{2} P / \partial T^{2}\right) \cdot d \rho / \rho^{2} .
$$

Figure 2 shows the well-known zero slope and curvature of the critical isotherm at the critical point.
This monotonic behavior (nonnegative slopes) is difficult to achieve from equations of state, yet is very important for such computations as -

$$
\begin{gathered}
C_{p}(\rho, T)=C_{v}(\rho, T)+T \cdot(\partial P / \partial T)^{2} /(\partial P / \partial \rho) / \rho^{2}, \\
W(\rho, T)=\left[C_{p} \cdot(\partial P / \partial \rho) / C_{v}\right]^{1 / 2} .
\end{gathered}
$$

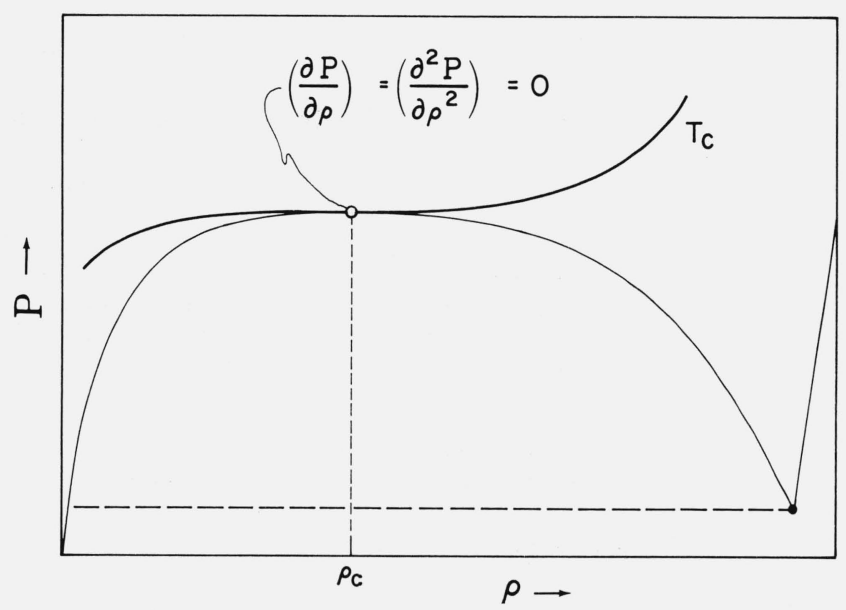

Figure 2. Behavior of the critical isotherm.

The liquid-vapor envelope, $T_{\sigma}(\rho)$, figure 3 , is an important boundary of the $P(\rho, T)$ surface for the equation of state. We constrain the equation to this bound-

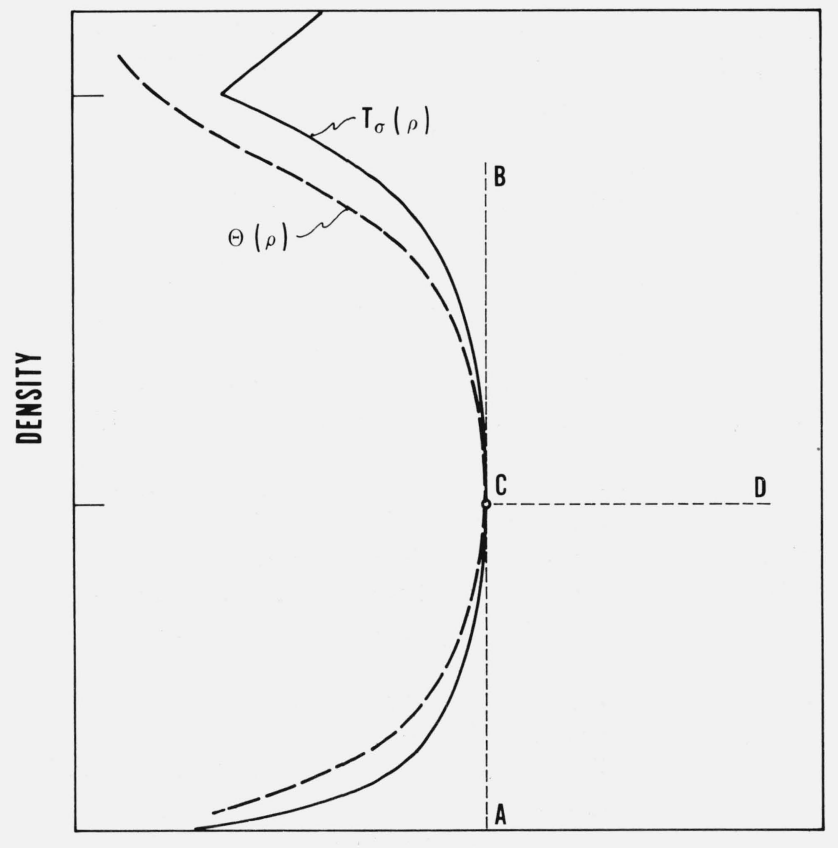

TEMPERATURE

Figure 3. Behavior of the locus $\theta(\rho)$.

Point $C$ is the critical point, and $T_{\sigma}(\rho)$ is the liquid-vapor coexistence envelope 
ary as follows, by use of the vapor pressures and the coexistence temperatures $T_{\sigma}(\rho)$ formulated in the appendix.

For any density (isochore), obtain the coexistence temperature from the function $T_{\sigma}(\rho)$. Use this to obtain the vapor pressure $P_{\sigma}\left[T_{\sigma}(\rho)\right]$ as a function of density, thus defining the equation of state at coexistence. By subtraction one then obtains an equation of the type of eq (1). The melting line is not a part of this equation of state

$$
P-P_{\sigma}(\rho)=\rho R \cdot\left[T-T_{\sigma}(\rho)\right]+\rho^{2} R T_{c} \cdot F(\rho, T),
$$

where,

$$
F(\rho, T) \equiv B(\rho) \cdot \Phi(\rho, T)+C(\rho) \cdot \Psi(\rho, T) .
$$

The functions $B(\rho)$ and $C) \rho$ ) are polynomial coefficients to be found by least squares. The temperaturedependent functions $\Phi(\rho, T)$ and $\Psi(\rho, T)$ must be defined to be zero on the coexistence boundary at $T=T_{\sigma}(\rho)$, as shown by figures 4 and 5 .

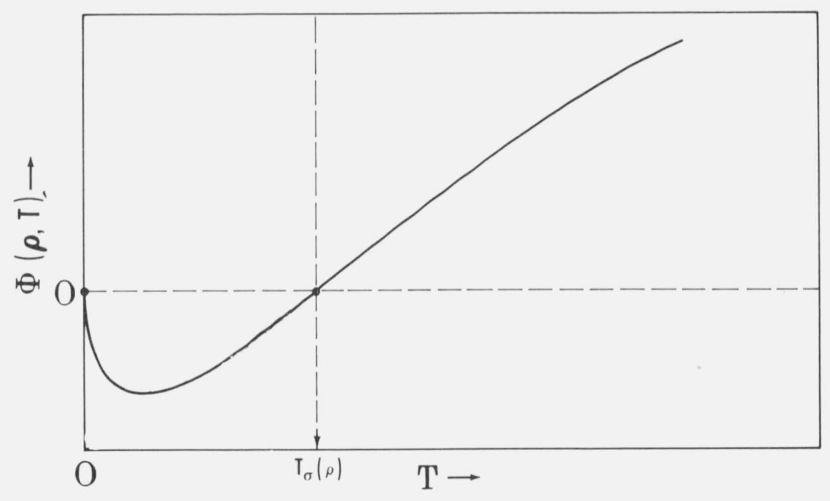

Figure 4. Behavior of the function $\Phi(\rho, \mathrm{T})$.

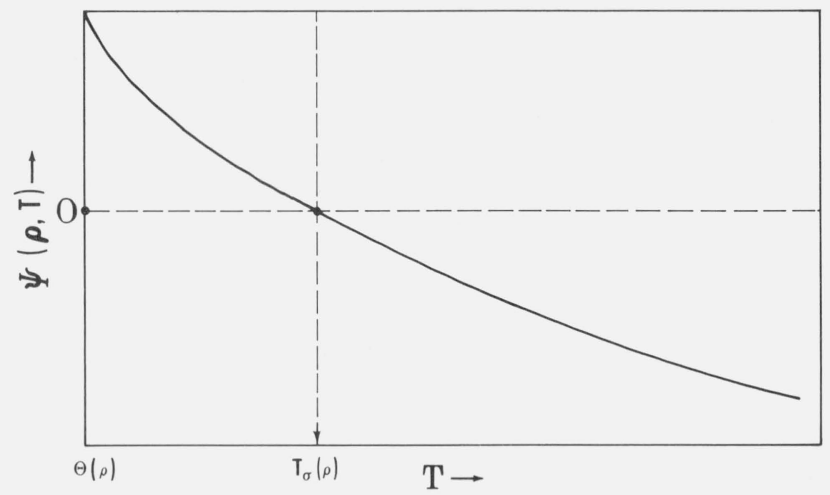

Figure 5. Behavior of the function $\Psi(\rho, \mathrm{T})$.

Equation (1) has, in addition to $\rho R T$, only two temperature-dependent functions, which is the minimum number of functions (monotonic in $T$ ) needed to describe the sigmoid shape of isochores in the density range $\rho_{c}<\rho<2 \cdot \rho_{c}$, figure 1 .

The first of these functions is -

$$
\Phi(\rho, T) \equiv x^{1 / 2} \cdot \ln [u(\rho, T)],
$$

where $u(\rho, T) \equiv T / T_{\sigma}(\rho)$. It is linear $\left(\partial^{2} \Phi / \partial T^{2}=0\right)$ everywhere on the coexistence boundary at $u=1$, figure 4. It therefore gives a critical isochore which is linear at the critical point because $C(\rho)=0$ by definition along this isochore, eq (6).

The second of these functions, $\Psi(\rho, T)$, is defined such that eq (1) will yield a maximum in the specific heats $C_{v}(\rho, T)$ at the critical point via eq (a). We first define the arguments -

$$
\begin{aligned}
\omega(\rho, T) & \equiv \delta \cdot[T / \theta(\rho)-1] . \\
\omega_{\sigma}(\rho) & \equiv \delta \cdot\left[T_{\sigma}(\rho) / \theta(\rho)-1\right],
\end{aligned}
$$

where $\delta$ is an arbitrary constant, and $\theta(\rho)$ is our locus of temperatures inside the coexistence envelope, figure 3 ,

$$
\theta(\rho) \equiv T_{\sigma}(\rho) \cdot \exp [-\alpha \cdot f(\sigma)] .
$$

The function $f(\sigma)$ here is normalized to unity at the liquid triple-point density -

$$
f(\sigma) \equiv|\sigma-1|^{3} /\left(\sigma_{t}-1\right)^{3},
$$

where $\sigma_{t} \equiv d_{t} / d_{c}$ is a constant.

Function $\Psi(\rho, T)$ now is defined as the difference,

$$
\Psi(\rho, T) \equiv \psi(\rho, T)-\psi_{\sigma}(\rho),
$$

such that $\Psi=0$ at coexistence, $T=T_{\sigma}(\rho)$. Component functions, $\psi(\rho, T)$, are designed to give infinite curvature $\left(\partial^{2} \psi / \partial T^{2}\right)$ at the origin, $\omega=0$,

$$
\begin{gathered}
\psi_{1}(\rho, T) \equiv[1-\omega \cdot \ln (1+1 / \omega)] / x, \\
\psi_{\sigma}(\rho) \equiv\left[1-\omega_{\sigma} \cdot \ln \left(1+1 / \omega_{\sigma}\right)\right] / x_{\sigma} .
\end{gathered}
$$

Figure 5 shows behavior of $\Psi(\rho, T)$. Sufficiently far away from the critical point it behaves roughly like $1 / T^{2}$, found in the well-known, Beattie-Bridgeman equation of state.

Behavior of the coefficients $B(\rho)$ and $C(\rho)$ in eq (1) is shown by figure 6 for methane. The following polynomial representations have been developed tediously by trial,

$$
\begin{aligned}
& B(\rho) \equiv B_{0}+B_{1} \cdot \rho+B_{2} \cdot \rho^{2} /\left(1+b \cdot \rho^{2}\right), \\
& C(\rho) \equiv(\sigma-1) \cdot\left(\sigma-C_{0}\right) \cdot\left(C_{1}+C_{2} \cdot \rho\right) .
\end{aligned}
$$

The sign of the curvature of isochores $\left(\partial^{2} P / \partial T^{2}\right)$ at the coexistence boundary is determined uniquely by the sign of $C(\rho)$, figure 6 , because $\Phi(\rho, T)$ is linear 

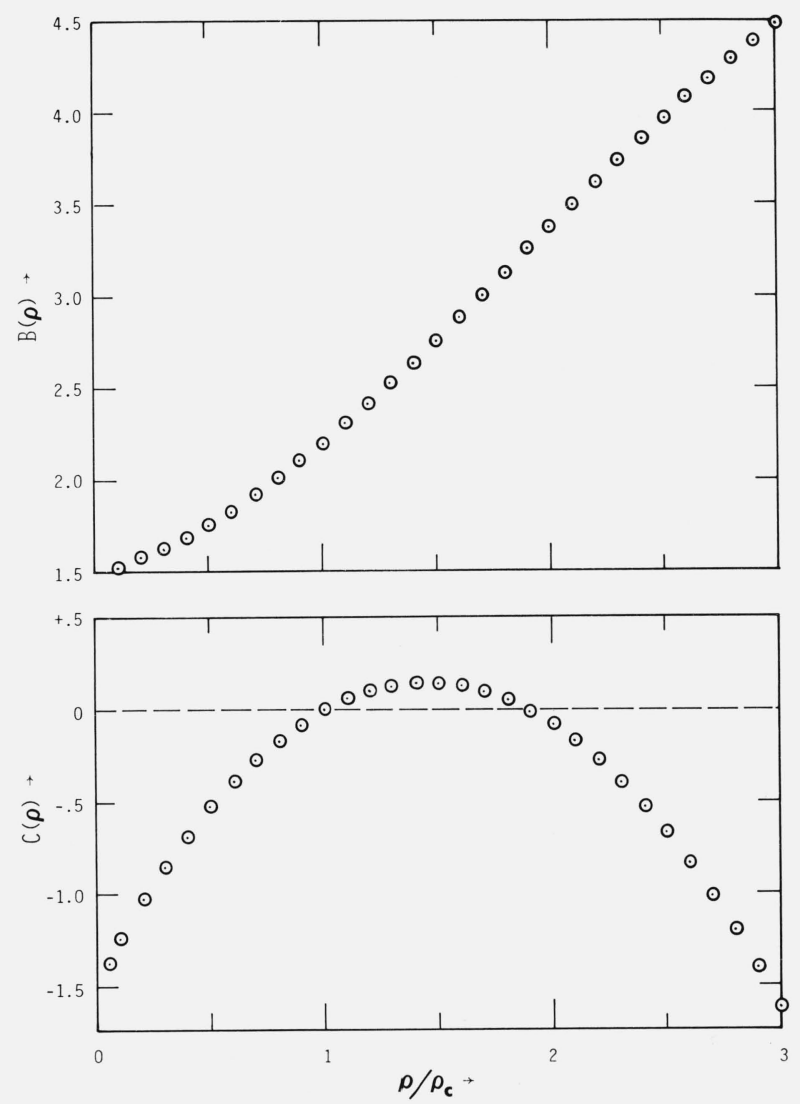

Figure 6. Behavior of the coefficients $\mathrm{B}(\rho), \mathrm{C}(\rho)$ for methane.

on this boundary. The root in $C(\rho)$ at $\sigma=1.9$ was found by least squares for methane and for ethane. It then was introduced as the constant $C_{0}$ in eq (6). This constraint is valuable because, under various conditions, we often have failed to obtain any such root from $P V T$ data by least squares.

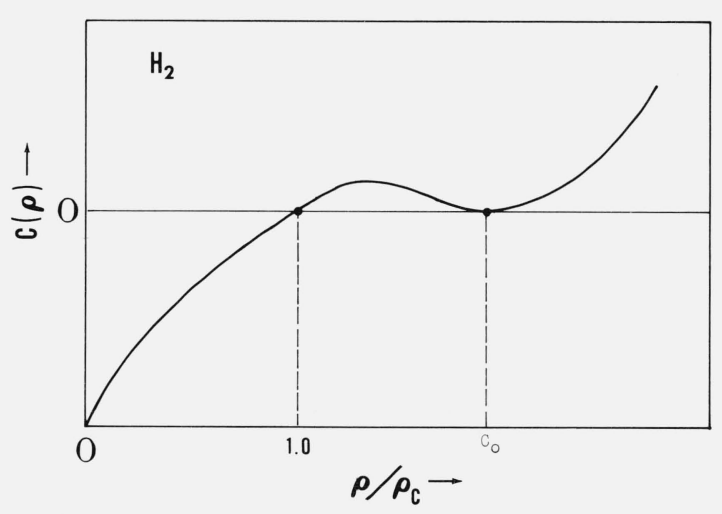

FiguRE 7. Presumed behavior of C $(\rho)$ for hydrogen, reflecting observed positive isochore curvatures in compressed liquid states at the lowest temperatures [7].
Figure 7 shows the presumed behavior of $C(\rho)$ for hydrogen, needed to give the observed positive curvature of isochores in compressed liquid states at the lowest temperatures $[3,7]$. The behavior of figure 7 is described by a double root at $C_{0}$, e.g.,

$$
C(\rho)=C_{1} \cdot(\sigma-1) \cdot\left(\sigma-C_{0}\right)^{2},
$$

or without a root by-

$$
C(\rho)=(\sigma-1) \cdot\left(C_{1}+C_{2} \cdot \rho+C_{3} \cdot \rho^{2}\right) .
$$

Table 2 presents constants of eq (1) for methane and ethane. Individual deviations of experimental PVT data from eq (1) are given for ethane in [15]. For methane with eq (1) they are comparable with those obtained in [14] using nine least-squares coefficients. Complete computer programs are reported in [15] for adjusting the vapor-pressure equation, the orthobaric-densities equations, the coexistence-temperatures equations, and the equation of state (1).

TABLE 2. Constants for equation of state (1)

\begin{tabular}{l|c|cc}
\hline \hline & Methane & \multicolumn{2}{|c}{ Ethane } \\
\hline$\alpha$ & 2 & 2 & \\
$b$ & 1 & 1 & \\
$\delta$ & $1 / 2$ & 1.9 & \\
$C_{0}$ & 1.9 & 1.848167996 \\
$B_{0}$ & 1.508212989 & 1.569704511 \\
$B_{1}$ & 0.654490304 & 5.560186452 \\
$B_{2}$ & 4.132082291 & & \\
$C_{1}$ & -0.765409076 & -1.042842462 \\
$C_{2}$ & -0.059088717 & +0.224978299 \\
$N$ & 756 & 562 & \\
$\Delta P / P, \%$ & 0.42 & 0.57 & \\
\hline
\end{tabular}

\section{Comments on the Equation}

Behavior of the critical isotherm from eq (1) at the critical point is deduced as follows. The functions $P_{\sigma}[T \sigma(\rho)]$ and $\Phi(\rho, T)$ depend directly upon $T_{\sigma}(\rho)$, which gives the overpowering factor $\exp [-\gamma|| \sigma-1 \mid]$ for derivatives with respect to density at the critical point, $\sigma=1$. The function $\Psi(\rho, T)$ has a finite third derivative because it depends also on $\theta(\rho)$, eq (3). Its coefficient, $C(\rho)$, however, is zero at the critical density, eq (6). The first, second, and third derivatives of eq (1) therefore are zero at the critical point.

Detailed examinations of this isotherm from eq (1) show, however, that small variations in the assigned $\left(\rho_{c}, T_{c}\right)$ critical point give small irregularities (negative slopes) nearby at $\sigma \lessgtr 1$. We find that, given an accurate value of $T_{c}$, eq (1) serves to find the critical density which yields a well-behaved critical isotherm [15]. For methane and ethane the value of the critical density obtained by this method is roughly 1 percent lower than estimated by the conventional procedure of extrapolating the rectilinear diameter to the critical 
temperature $[14,15,20]$, but in each case falls within bounds of uncertainty in published works.

Ioschore inflection points figure 1, calculated from eq (1), are obtained as the difference of second derivatives (versus $T$ ) from the functions composing $F(\rho, T)$. We therefore expect high sensitivity to the analytical forms of $\Phi(\rho, T)$ and $\Psi(\rho, T)$. Variations of these forms might improve accuracy in representing $P V T$ data. In the following we describe two alternative functions for $\Psi(\rho, T)$ from among many different functions investigated both for $\Phi(\rho, T)$ and for $\Psi(\rho, T)$. We then compare computed specific heats obtained via these two forms in the modified equation of state described below. Each of the following component functions must be differenced, as indicated by eq (4), to obtain $\Psi(\rho, T)$ for the equation of state. The argument for each of the following functions is changed to -

$$
\omega(\rho, T) \equiv[1-\theta(\rho) / T] .
$$

In (7) the adjustable exponent is $1<\epsilon<2$, and the adjustable coefficient was selected by trial to be $\delta=1 / \epsilon$,

$$
\psi_{2}(\rho, T) \equiv 1-\left(\omega-\delta \cdot \omega^{\epsilon}\right) /(1-\delta) .
$$

This function approaches zero at high temperatures in proportion to $1 / T^{2}$, as seen by expanding $\omega^{\epsilon}$. By trial with methane and ethane $P V T$ data, we found $\epsilon=3 / 2$, and hence $\partial^{2} \psi_{2} / \partial T^{2}$ behaves like $(1 / \omega)^{1 / 2}$ on approach to the origin, $\omega=0$.

In (8) we use the arbitrary constant $0 \leqslant \delta \leqslant 1$ to give relative weighting to two terms behaving, respectively, like $1 / T$ and $1 / T^{2}$ at high temperatures,

$$
\psi_{3}(\rho, T) \equiv \delta \cdot(\theta / T)+(1-\delta) \cdot[1-\omega+\omega \cdot \ln (\omega)] .
$$

The last term in (8) was explored for hydrogen [10]. Equation (8) can be simplified for computations. This function gives $\partial^{2} \psi_{3} / \partial T^{2} \sim(1 / \omega)$ on approach to the origin, $\omega=0$, similar to definition (4-a) above.

With above functions, we find that coefficients $B_{1}$ and $C_{2}$ of eq (1) become nonsignificant for methane, leaving an equation of state with only three arbitrary, least-squares coefficients.

For computations on mixtures, the simplest possible equation of state is desirable. We have modified (1) such that it is suitable for corresponding-states adaptations, by specifying that densities shall be reduced at the critical point (hence $R=0.0831434 \cdot d_{c}, \mathrm{bar} / \mathrm{K}$ ), and by introducing the critical pressure. The functions $\Phi(\rho, T)$ and $\Psi(\rho, T)$ remain unchanged-

$$
P-P_{\sigma}(\rho)=\sigma R \cdot\left[T-T_{\sigma}(\rho)\right]+P_{c} \cdot \sigma^{2} \cdot F(\rho, T),
$$

$$
B(\sigma) \equiv B_{0}+B_{2} \cdot \sigma^{2} /\left(1+b \cdot \sigma^{2}\right),
$$

$$
C(\sigma) \equiv C_{1} \cdot(\sigma-1) \cdot\left(\sigma-C_{0}\right) .
$$

Table 3 for methane gives the rms of relative density deviations for authors in [14], corresponding to each

\begin{tabular}{|c|c|c|c|c|}
\hline \multirow{2}{*}{\multicolumn{2}{|c|}{$\begin{array}{l}\text { Equation of state .......... } \\
\text { Function } \psi(\rho, T) \ldots \ldots \ldots .\end{array}$}} & \multirow{2}{*}{$\frac{\text { eq }(1)}{\psi_{1}, \text { eq }(4-a)}$} & \multicolumn{2}{|c|}{ eq $(1-A)$} \\
\hline & & & $\psi_{2}$, eq $(7)$ & $\psi_{3}$, eq $(8)$ \\
\hline Authors & $N$ & & & \\
\hline Virial eq, $[14] \ldots \ldots \ldots$ & 46 & 0.06 & 0.12 & 0.16 \\
\hline Douslin et al. $[4] \ldots .$. & 171 & .06 & .13 & .10 \\
\hline NBS [14] .............. & 539 & .51 & .60 & .50 \\
\hline Mean $\Delta P / P, \% \ldots$ & 756 & .42 & .46 & .45 \\
\hline
\end{tabular}
function $\psi(\rho, T)$ described above and, on the bottom line, the mean of combined pressure deviations. Tables 4 and 5 give the constants for eq (1-A), so that

\begin{tabular}{|c|c|c|}
\hline & Methane & Ethane \\
\hline$b$ & $1 / 8$ & $1 / 8$ \\
\hline$\alpha$ & $1 / 2$ & $1 / 2$ \\
\hline$\delta$ & $2 / 3$ & $2 / 3$ \\
\hline$\epsilon$ & $3 / 2$ & $3 / 2$ \\
\hline$B_{0}$ & 1.989421671 & 2.237356347 \\
\hline$B_{2}$ & 0.792435706 & 0.930497491 \\
\hline$C_{0}$ & 1.9 & 1.9 \\
\hline$C_{1}$ & -0.830940825 & -1.049411810 \\
\hline & 756 & 562 \\
\hline$\Delta P / P, \%$ & 0.46 & 0.79 \\
\hline
\end{tabular}
it will be possible to compute specific heats.

TABLE 3. Methane density deviations, rms percent

TABLE 4. Constants for equation of state $(1-A)$, using $\psi_{2}(\rho, \mathrm{T})$, $e q(7)$

TABLE 5. Constants for equation of state $(1-A)$, using $\psi_{3}(\rho, \mathrm{T})$, $e q(8)$

\begin{tabular}{l|l|l}
\hline \hline & Methane & \multicolumn{2}{|c}{ Ethane } \\
\hline$b$ & $1 / 8$ & $1 / 8$ \\
$\alpha$ & $1 / 2$ & $2 / 3$ \\
$\delta$ & $1 / 3$ & $1 / 2$ \\
$B_{0}$ & 1.979455787 & 2.180554662 \\
$B_{2}$ & 0.801724817 & 0.981453571 \\
$C_{0}$ & 1.9 & 1.9 \\
$C_{1}$ & -0.946570360 & -1.354333999 \\
$N$ & 756 & 562 \\
$\Delta P / P, \%$ & 0.45 & 0.75 \\
\hline
\end{tabular}

The interaction specific heats $\left(C_{v}-C_{v}^{0}\right)$ for methane have been compared along the critical isochore, as computed by equations (a) and (1-A), using the functions $\psi_{2}(\rho, T)$ and $\psi_{3}(\rho, T)$. Experimental behavior of specific heats suggests that these data should be described very near the critical temperature by-

$$
\left(C_{v}-C_{v}^{0}\right) \sim\left(T / T_{c}-1\right)^{-n},
$$


where exponent $0<n<1$, and usually, $0.05 \leqslant n \leqslant 0.15$, [16].

Figure 8 shows the computed results via $\psi_{2}(\rho, T)$, eq (7). Their qualitative behavior is correct, but they cannot be represented by (9). Instead, they are described accurately to $210 \mathrm{~K}$ by -

$\left(C_{v}-C_{v}^{0}\right)=15.1-18.0 \cdot\left(T / T_{c}-1\right)^{1 / 3}, \quad J / \mathrm{mol} / K$, suggesting that they might become finite at the critical temperature.
Using $\psi_{3}(\rho, T)$, eq (8), on the other hand, yields results on the logarithmic plot of figure 9. The slope, $n \approx 1 / 3$, is close to that observed for our experimental oxygen specific heats [8].

We conclude that $\psi_{2}(\rho, T)$ gives too weak a curvature to isochores from (1-A) approaching coexistence, and that the forms $\psi_{1}(\rho, T)$ and $\psi_{3}(\rho, T)$ may be preferable, despite the logarithmic infinity in their first derivatives versus $T$ at the origin, $\omega=0$.

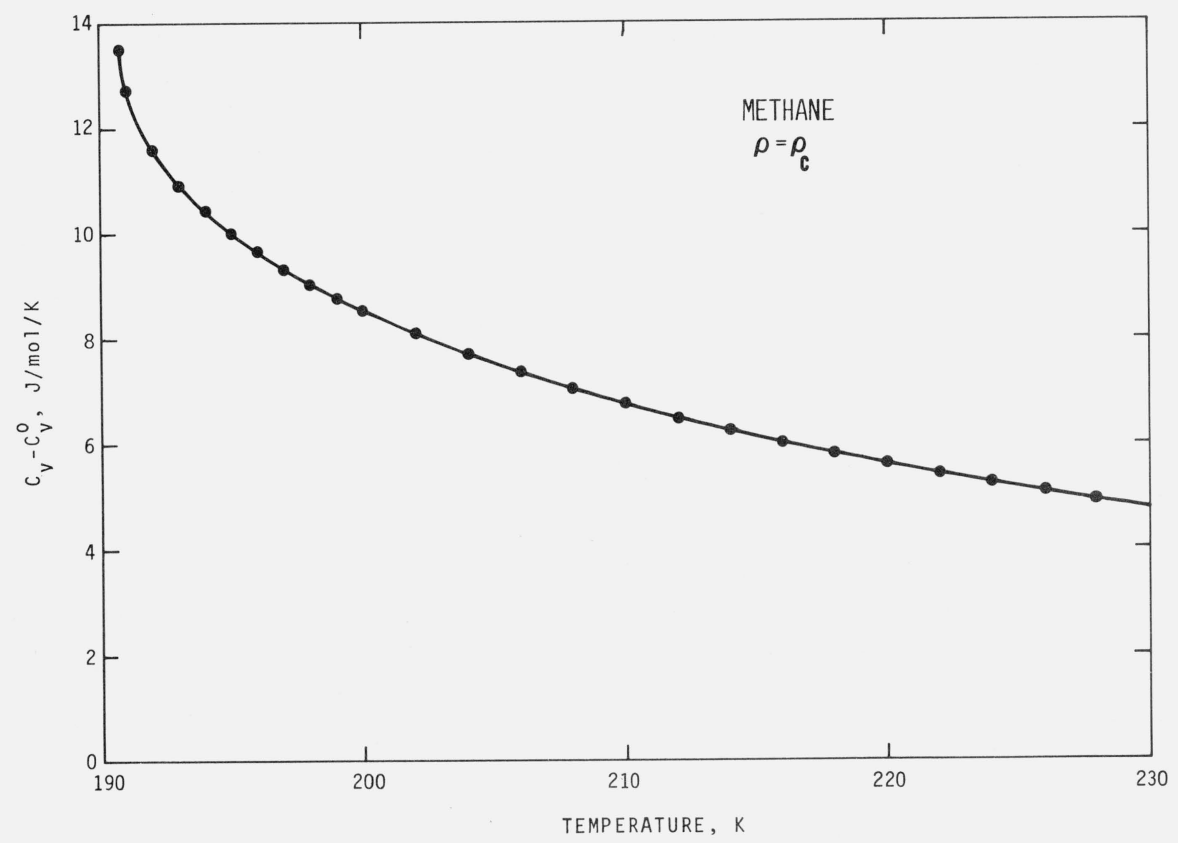

FIGURE 8. Computed interaction specific heats $\left(\mathrm{C}_{\mathrm{v}}-\mathrm{C}_{\mathrm{v}}^{0}\right)$ of methane along the critical isochore, via eq $(1-\mathrm{A})$ and $\psi_{2}(\rho, \mathrm{T})$.

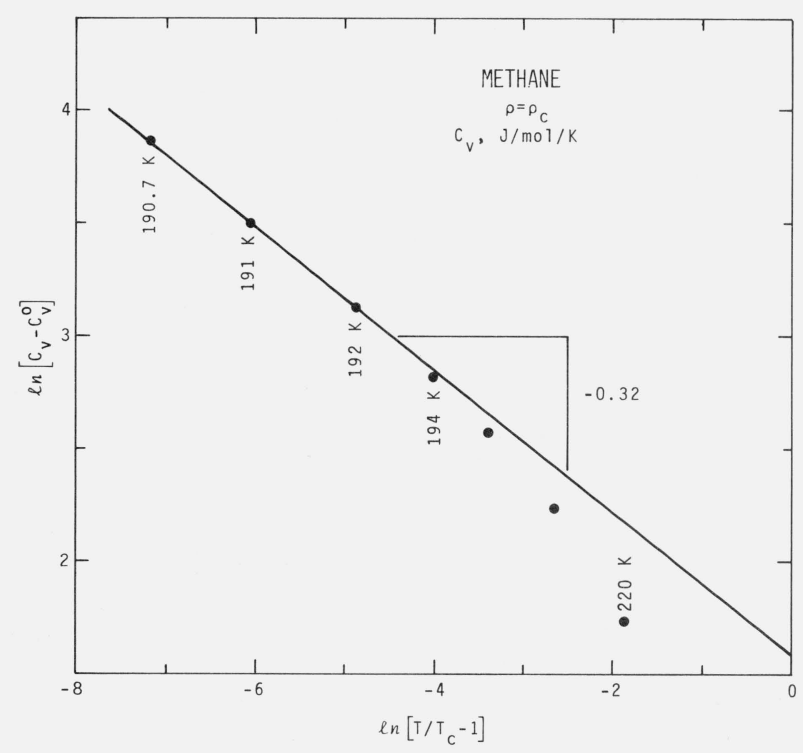

Figure 9. Computed interaction specific heats $\left(\mathrm{C}_{\mathrm{v}}-\mathrm{C}_{\mathrm{v}}^{0}\right)$ of methane along the critical isochore, via eq $(1-\mathrm{A})$ and $\psi_{3}(\rho, \mathrm{T})$.

\section{Conclusions}

As argued above, the present equation of state (1) is rational because we understand the purpose of most of its component parts. Inclusion of the vapor pressures and orthobaric densities in this equation merely incorporates these physical properties which almost invariably must be used for a consistent network of thermodynamic functions, and eliminates the long-standing problem of continuity at the coexistence boundary [26].

We believe eq (1) to be valuable for thermal computations because it ensures an inherently correct behavior of the $P(\rho, T)$ surface, giving a maximum in the specific heats $C_{v}(\rho, T)$ at the critical point. The very small number of arbitrary least-squares coefficients (five, and possibly only three) facilitates comparisons of the equation of state for different substances, and may be attractive for work on mixtures.

We cannot expect eq (1) to represent some highprecision $P V T$ data as well as equations with a much greater number of arbitrary constants. As the precision of "good" $P V T$ data probably is often much bet- 
ter than absolute accuracy, especially in the critical region, it would appear to be a self-defeating exercise to strive for the ultimate "representation" while ignoring essential features of the $P(\rho, T)$ surface. Any inaccuracies in the given, liquid-vapor, $P-\rho-T$ boundary for eq (1), however, will be propagated along calculated isochores because eq (1) originates on this boundary.

Equation (1) almost certainly cannot be integrated analytically to express derived properties in closed form. It therefore would not be convenient for multiproperty analysis [2]. Equations amenable to integration, however, probably are not accurate in the critical region, which influences a large fraction of the $P(\rho, T)$ surface. To some extent, this must create a need for multiproperty analysis.

For methane with an equation of state similar to eq (1), on the other hand, we have used a minimum of specific heat data only to compute around the critical point for high densities near the critical temperature (fig. 3). In all other regions we computed specific heats and speeds of sound "a priori" via ideal gas specific heats and the equation of state, finding acceptable agreement with experimental data. We concluded that if such data do not exist experimentally, they could be estimated via the present type of equation of state for many but probably not all regions of the $P(\rho, T)$ surface [14].

\section{Appendices}

\subsection{The Vapor Pressure Equation}

The original form of our vapor pressure function [9] is satisfactory for methane [14], but for ethane it has been necessary to add the term $d \cdot x^{4}$ [15]. Define the argument -

$$
x(T) \equiv\left(1-T_{t} / T\right) /\left(1-T_{t} / T_{c}\right),
$$

when the function is -

$$
\ln \left(P / P_{t}\right)=a \cdot x+b \cdot x^{2}+c \cdot x^{3}+d \cdot x^{4}+e \cdot x \cdot(1-x)^{3 / 2} .
$$

Table 6 presents the coefficients.

TABLE 6. Constants for vapor-pressure equation (5.1)

\begin{tabular}{l|r|r}
\hline \hline & \multicolumn{1}{|c|}{ Methane } & \multicolumn{1}{|c}{ Ethane } \\
\hline$a$ & 4.77748580 & 10.79549166 \\
$b$ & 1.76065363 & 8.35899001 \\
$c$ & -0.56788894 & -3.11490770 \\
$d$ & 0.0 & -0.64969799 \\
$e$ & 1.32786231 & 6.07349549 \\
\hline
\end{tabular}

\subsection{The Orthobaric Densities}

The following expressions are constrained to the boundaries, the triple- and critical points [15]. In each case the basic behavior is described by-

$$
Y(\rho, T)=\text { const. }
$$

and polynomials are selected to represent small deviations. For the saturated liquid, define the variables,

$$
\begin{aligned}
x(T) & \equiv\left(T_{c}-T\right) /\left(T_{c}-T_{t}\right), \\
y(\rho) & \equiv\left(\rho-\rho_{c}\right) /\left(\rho_{t}-\rho_{c}\right), \\
Y(\rho, T) & \equiv(y-x) /\left(x^{\epsilon}-x\right),
\end{aligned}
$$

when the function is -

$$
Y=a+b \cdot x^{2 / 3}+c \cdot x .
$$

For the saturated vapor, define the variables,

\begin{tabular}{|c|c|c|}
\hline & Methane & Ethane \\
\hline \multicolumn{3}{|c|}{ Saturated liquid densities, eq (5.2) } \\
\hline$\epsilon$ & 0.36 & 0.33 \\
\hline$a$ & .85953758 & .72190944 \\
\hline$b$ & .02436448 & .29657790 \\
\hline$c$ & -.02685285 & -.30036548 \\
\hline \multicolumn{3}{|c|}{ Saturated vapor densities, eq (5.3) } \\
\hline$\epsilon$ & 0.41 & 0.39 \\
\hline$A_{1}$ & .41714211 & .21587515 \\
\hline$A_{2}$ & -.51949762 & -.08522342 \\
\hline$A_{3}$ & 1.20777553 & -.61523457 \\
\hline$A_{4}$ & -1.46130509 & .25452490 \\
\hline$A_{5}$ & 0.57658540 & .15177230 \\
\hline
\end{tabular}

$$
\begin{aligned}
x(T) & \equiv\left(T_{c} / T-1\right) /\left(T_{c} / T_{t}-1\right), \\
y(\rho) & \equiv \ln \left(\rho_{c} / \rho\right) / \ln \left(\rho_{c} / \rho_{t}\right) \\
Y(\rho, T) & \equiv(y-x) /\left(x^{\epsilon}-x\right),
\end{aligned}
$$

when the function is -

$$
Y=A_{1}+\sum_{i=2}^{5} A_{i} \cdot x^{i / 3} .
$$

Table 7 presents constants for (5.2) and (5.3).

TABLE 7. Constants for orthobaric densities equations

\subsection{The Coexistence Envelope, $\mathrm{T}_{\sigma}(\boldsymbol{\rho})$}

This envelope is shown by figure 3 . For the equation of state (1) we obtain the coexistence temperature for any density from the following analytical descriptions. If the coexistence density must be found at a given temperature, it is obtained (for consistency in the equation of state) from the following expressions by iteration, using eqs (5.2) and (5.3) only to estimate an initial density.

An important feature of the present formulations $[15]$ is that derivatives -

$$
d^{n} T \sigma / d p^{n}
$$

of all orders, $n$, are zero at $\sigma=1$, the critical point. We describe $T_{\sigma}(\rho)$ in two parts, according as $\sigma \lessgtr 1$. 
This simplifies constraint to the boundaries (liquid and vapor triple points). For each range the dependent variable is -

$$
Y\left[T_{\sigma}(\rho)\right] \equiv\left(T_{c} / T_{\sigma}-1\right) /\left(T_{c} / T_{t}-1\right),
$$

and we use the following function, infinite at the critical density

$$
U(\sigma) \equiv-\gamma \cdot\left[1 /|\sigma-1|-1 /\left|\sigma_{t}-1\right|\right]
$$

where $\sigma_{t}=d_{t} / d_{c}$ is a constant, and $d_{t}$ refers to vapor or liquid at the triple point according as $\sigma \lessgtr 1$.

For the liquid range at $\sigma \geqslant 1$ the equation is -

$$
\ln (Y)=U(\sigma)+\sum_{i=1}^{5} A_{i} \cdot\left(\sigma^{i}-\sigma_{t}^{i}\right)
$$

For extremely low densities in the vapor range at $\sigma \leqslant 1$ we modify the above expression as follows. Define the variable

$$
W(\sigma) \equiv \ln (1+s / \sigma) / \ln \left(1+s / \sigma_{t}\right),
$$

where $s$ is an arbitrary constant. Our equation for the vapor range now is -

$$
\begin{aligned}
\ln (\bar{Y})= & U(\sigma)+B_{0} \cdot \ln (W)+B_{1} \cdot\left(\sigma^{1 / 3}-\sigma_{t}{ }^{1 / 3}\right) \\
& +B_{2} \cdot\left(\sigma^{2 / 3}-\sigma_{t}^{2 / 3}\right)+\sum_{i=3}^{7} B_{i} \cdot\left(\sigma^{i-2}-\sigma_{t}^{i-2}\right) .
\end{aligned}
$$

Table 8 presents constants for (5.4) and (5.5)

TABLE 8. Constants for the $\mathrm{T}_{\sigma}(\rho)$ equations

\begin{tabular}{l|l|l}
\hline \hline & Methane & Ethane \\
\hline
\end{tabular}

Saturated liquid, eq (5.4)

\begin{tabular}{l|rl|rl}
\hline$\gamma$ & \multicolumn{1}{|c|}{$1 / 2$} & & \multicolumn{1}{|c}{$1 / 2$} & \\
$A_{1}$ & 11.4317 & 7230 & 23.7245 & 1840 \\
$A_{2}$ & -3.8765 & 9480 & -14.8860 & 5161 \\
$A_{3}$ & 0.5378 & 8326 & 5.4317 & 7443 \\
$A_{4}$ & .0 & & -1.0715 & 0566 \\
$A_{5}$ & .0 & & 0.0913 & 5183 \\
\hline
\end{tabular}

Saturated vapor, eq (5.5)

\begin{tabular}{l|rl|rl}
\hline$s$ & \multicolumn{1}{|c|}{$1 / 4$} & & \multicolumn{1}{|l}{$1 / 4$} & \\
$B_{0}$ & 0.9034 & 9557 & 0.8681 & 0517 \\
$B_{1}$ & .0 & & .0151 & 6978 \\
$B_{2}$ & \multicolumn{1}{|c}{.0} & & -.7296 & 0432 \\
$B_{3}$ & -.3834 & 4338 & 1.0096 & 5493 \\
& & & & \\
$B_{4}$ & -3.9210 & 8638 & -8.7340 & 2710 \\
$B_{5}$ & 6.2600 & 3837 & 21.1071 & 2823 \\
$B_{6}$ & -9.3296 & 0083 & -31.4499 & 4087 \\
$B_{7}$ & 5.6060 & 2816 & 17.8637 & 0397 \\
\hline
\end{tabular}

Anneke L. Sengers emphasized to us some time ago the importance of the critical region for the entire equation of state, thus motivating present developments. In this laboratory, R. D. McCarty provided the essential least-squares program, and we are indebted to D. E. Diller and L. A. Weber for discussions and valuable suggestions. The American Gas Association very kindly has supported this work.

\section{References}

[1] Bazúa, E. R., and Prausnitz, J. M., Vapor-liquid equilibria for cryogenic mixtures, Cryogenics 11, No. 2, 114 (April, 1971).

[2] Cox, K. W., Bono, J. L., Kwok, Y. C., and Starling, K. E., Multiproperty analysis. Modified BWR equation for methane from PVT and enthalpy data, Ind. Eng. Chem. Fundam. 10, No. 5, 245(1971).

[3] Diller, D. E., The specific heats $\left(C_{v}\right)$ of dense simple fluids, Cryogenics 11, No. 3, 186 (June, 1971).

[4] Douslin, D. R., Harrison, R. H., Moore, R. T., and McCullough, J. P., P-V-T relations for methane, J. Chem. Eng. Data 9, No. 3, 358 (1964).

[4a] Fisher, G. D. and Leland, T. W., Jr., Corresponding states principle using shape factors, Ind. Eng. Chem. Fundam. 9, No. 4, 537 (1970).

[5] Furtado, André W., The measurement and prediction of thermal properties of selected mixtures of methane, ethane, and propane, $(\mathrm{Ph}$. D. Thesis, Dept. of Chemical Engineering, Univ. of Michigan, Ann Arbor, Mich., Dec. 1973.)

[6] Goodwin, R. D., Approximate Wide-Range Equation of State for Parahydrogen, Advances in Cryogenic Engineering 6, 450 (Plenum Press, New York, 1961).

[7] Goodwin, R. D., An equation of state for fluid parahydrogen from the triple point to $100{ }^{\circ} \mathrm{K}$ at pressures to 350 atmospheres, J. Res. Nat. Bur. Stand. (U.S.) $71 \mathbf{A}$ (Phys. and Chem.), No. 3, 203-212 (May-June 1967).

[8] Goodwin, R. D. and Weber, L. A., Specific heats $C_{v}$ of fluid oxygen from the triple point to $300 \mathrm{~K}$ at pressures to 350 atmospheres, J. Res. Nat. Bur. Stand. (U.S.), 73A (Phys. and Chem.), No. 1, 15-24 (Jan.-Feb. 1969).

[9] Goodwin, R. D., Nonanalytic vapor pressure equation with data for nitrogen and oxygen, J. Res. Nat. Bur. Stand. (U.S.) 73A (Phys. and Chem.), No. 5, 487-491 (Sept.-Oct. 1969).

[10] Goodwin, R. D., Formulation of a nonanalytic equation of state for parahydrogen, J. Res. Nat. Bur. Stand. (U.S.) 73A (Phys. and Chem.), No. 6, 585-591 (Sept.-Oct. 1969).

[11] Goodwin, R. D., Estimation of critical constants $T_{c}, \rho_{c}$, from the $\rho(T)$ and $T(\rho)$ relations at coexistence, J. Res. Nat. Bur. Stand. (U.S.) 74A (Phys. and Chem.), No. 2, 221-227 (Mar.Apr. 1970).

[12] Goodwin, R. D. and Prydz, R., Densities of compressed liquid methane, and the equation of state, J. Res. Nat. Bur. Stand. (U.S.), (Phys. and Chem.), No. 2, 81-101 (Mar.-Apr. 1972).

[13] Goodwin, R. D., Nonanalytic equation of state for methane, constrained to the vapor-liquid $P-\rho-T$ boundary, unpublished NBS IR.

[14] Goodwin, R. D., The thermophysical properties of methane from 90 to $500 \mathrm{~K}$ at pressures to $700 \mathrm{bar}$, Nat. Bur. Stand. (U.S.), Tech. Note 653, 280 pages (Apr. 1974).

[15] Goodwin, R. D., Provisional values for the thermodynamic functions of ethane, unpublished manuscript, June, 1974.

[16] Green, M. S., and Sengers, J. V., Editors, Critical phenomena, Nat. Bur. Stand. (U.S.), Misc. Publ. 273, 256 pages (Dec. 1966).

[17] Kirk, B. S., Ziegler, W. T., and Mullins, J. C., A comparison of methods of predicting equilibrium gas phase compositions in pressurized binary systems containing an essentially pure condensed phase, Advances in Cryogenic Engineering 6, $413(1961)$

[18] Mollerup, J., and Rowlinson, J. S., The prediction of the densities of liquefied natural gas and of lower molecular weight hydrocarbons, Chem. Eng. Sci. 29, 1373 (1974). 
[19] Rowlinson, J. S., Molecular theories of liquids and mixtures, Ind. Eng. Chem. 59, No. 12, 28(1967).

[20] Rowlinson, J. S., Liquids and Liquid Mixtures, p. 98, (Butterworths Scientific Publications, London, 1959). See also second edition (1969).

[21] Smith, G. E., Sonntag, R. E., and Van Wylen, G. J., Analysis of the solid-vapor equilibrium system carbon dioxide-nitrogen, Advances in Cryogenic Engineering 8, 162 (1963).

[2la] Smith, W. R., Perturbation theory and one-fluid corresponding states theories for fluid mixtures, The Can. J. Chem. Eng. 50, 271 (1972).

[22] Sood, S. K. and Haselden, G. G., Prediction methods for vaporliquid equilibria in multicomponent cryogenic mixtures, Cryogenics 10, No. 3, 199 (June, 1970).

[23] Starling, K. E., Fluid Thermodynamic Properties for Light Hydrocarbon Systems (Gulf Publishing Co., Houston, Texas, 1973).
[24] Tsonopoulos, C. and Prausnitz, J. M., Equations of state: a review for engineering applications, Cryogenics 9, No. 5, 315 (Oct., 1969).

[25] Verbeke, O.B., Representation of $P-V-T$ data by means of a "universal" state equation for simple fluids, Paper F-6, Advances in Cryogenic Engineering 18, 256 (1972).

[26] Wagner, W., A method to establish equations of state exactly representing all saturated state variables applied to nitrogen, Cryogenics 12, No. 3, 214 (June, 1972).

[27] Zellner, M. G., Claitor, L. C., and Prausnitz, J. M., Prediction of vapor-liquid equilibria and enthalpies of mixtures at low temperatures, Ind. Eng. Chem. Fundam. 9, No. 4, 549 (1970).

[28] Zemansky, M. W., Heat and Thermodynamics (McGraw-Hill Book Co., Inc., New York, 1957).

(Paper 79A1-844) 